\title{
Standardising online clinical reasoning teaching for undergraduate students in Somaliland
}

\author{
Authors: Rebecca C Stout and Katherine Elliott
}

\begin{abstract}
Aims
In 2013, there was a shortage of over 7 million healthcare workers worldwide, with African and Asian nations hardest hit. It is being increasingly recognised that online platforms and e-learning can be a useful way in which to subsidise face-to-face teaching. One such programme is run through the King's Somaliland Project, in which King's College London works with two universities within Somaliland to deliver online tutorials to undergraduate students. The aim of this project was to standardise current tutorials delivered in order to begin quality assurance of teaching.
\end{abstract}

\section{Methods}

We have moved to standardise the process in order to ensure that all students have equal learning opportunities. Conditions which are not commonly seen in the UK, such as infectious diseases, have been removed from the clinical reasoning tutorials as it is believed that expertise for these conditions will be best among Somaliland tutors who see them regularly. Instead, topics selected by Somaliland tutors to include dyspnoea, seizure, stroke, upper gastrointestinal bleed and chest pain are taught. UK tutors are supplied with a presentation which is uploaded to the online platform and which works through increasingly complex cases. They are also supplied with a crib sheet which students are unable to view with common case-based scenarios.

\section{Results}

We have found that student attendance at tutorials has improved throughout this year, which may indicate that tutorials are more useful since they are now more standardised, with increased student participation. Feedback from tutors has also proved positive using this method of tutorial organisation.

\section{Conclusion}

We conclude the importance of taking into account expertise already available within a resource-poor setting so as to complement this. We also conclude the importance of ensuring that teaching is standardised within this setting. We are currently moving towards further quality assurance with peer observation of tutorials soon to be introduced.

Authors: King's College London, London, UK 